\title{
An Application of Skew Product Maps to Markov Chains
}

\author{
by
}

\author{
Zbigniew S. KOWALSKI
}

\section{Presented by Stanisław KWAPIEŃ}

Summary. By using the skew product definition of a Markov chain we obtain the following results:

(a) Every $k$-step Markov chain is a quasi-Markovian process.

(b) Every piecewise linear map with a Markovian partition defines a Markov chain for every absolutely continuous invariant measure.

(c) Satisfying the Chapman-Kolmogorov equation is not sufficient for a process to be quasi-Markovian.

0. Introduction. Consider a stationary Markov chain with finite state space, i.e., a probability space $(\Omega, P)$, a sequence of $\left(X_{n}\right)_{n=0}^{\infty}$ random variables $X_{n}: \Omega \rightarrow\{1, \ldots, s\}$ a probability vector $\vec{p}=\left(p_{1}, \ldots, p_{s}\right)$, a stochastic matrix $\Pi=\left(p_{i j}\right)_{s \times s}$ such that $\vec{p} \Pi=\vec{p}$ and

$$
P\left(X_{n+1}=j \mid X_{0}=i_{0}, \ldots, X_{n}=i_{n}\right)=P\left(X_{n+1}=j \mid X_{n}=i_{n}\right)=p_{i_{n} j} .
$$

To apply ergodic theory here, we need a sequence representation of the process. Therefore we assume that $\Omega=\{1, \ldots, s\}^{N}, N=\{0,1,2, \ldots\}, P$ is the measure given by

$$
P\left(\omega: \omega_{0}=i_{0}, \ldots, \omega_{n}=i_{n}\right)=p_{i_{0}} p_{i_{0} i_{1}} \cdots p_{i_{n-1} i_{n}}
$$

and $\sigma$ is the 1 -sided shift on $\Omega,(\sigma \omega)_{i}=\omega_{i+1}$. Finally, we get the measure preserving dynamical system $(\Omega, \mathcal{A}, P, \sigma, \alpha)$, where $\mathcal{A}$ is the $\sigma$-algebra generated by the cylinder sets $\left\{\omega: \omega_{i_{1}}=j_{1}, \ldots, \omega_{i_{n}}=j_{n}\right\}$ and $\alpha=\left\{A_{i}\right\}_{i=1}^{s}$, where $A_{i}=\left\{\omega: \omega_{0}=i\right\}, i=1, \ldots, s$. The process $\left(X_{n}^{\prime}\right)_{n=0}^{\infty}$ given by $X_{n}^{\prime}(\omega)=i \Leftrightarrow \sigma^{n}(\omega) \in A_{i}$ is a Markov chain with the same distribution

2000 Mathematics Subject Classification: Primary 37A05; Secondary 60J10.

Key words and phrases: skew product, Markov chain, quasi-Markovian process.

Research supported by grant MENII 1 P03A 021 29, Poland. 
as $\left(X_{n}\right)_{n=0}^{\infty}$. The above process is denoted by $(\sigma, \alpha)$ and called a Markov process.

For our aims we will use more general notations. Let $(X, \mathcal{A}, m, f, \alpha)$ be a measure preserving dynamical system where $(X, \mathcal{A}, m)$ is a probability Lebesgue space and additionally $f$ is a positively nonsingular map (i.e. $m(A)=0 \Rightarrow m(f(A))=0)$, and $\alpha=\left\{A_{i}\right\}_{i=1}^{s}$ is a generating partition, i.e. $\bigvee_{i=0}^{\infty} f^{-i} \alpha=\mathcal{A}$. We denote by $(f, \alpha)$ the process $\left(X_{n}\right)_{n=0}^{\infty}$ such that $X_{n}(x)=i \Leftrightarrow f^{n}(x) \in A_{i}$. For our aims it is convenient to use an equivalent definition of Bernoulli and Markov processes (see [4]). To this end let us consider another Lebesgue probability space $(Y, \mathcal{B}, q)$ and let $\left\{T_{i}\right\}_{i=1}^{s}$ be a family of positively and negatively nonsingular maps of $Y$ into $Y\left(T_{i}\right.$ is negatively nonsingular if $\left.q(B)=0 \Rightarrow q\left(T_{i}^{-1} B\right)=0\right)$. The process $(f, \alpha)$ and the family $\left\{T_{i}\right\}_{i=1}^{s}$ define the skew product map

$$
T(x, y)=\left(f(x), T_{a(x)}(y)\right),
$$

where $a: X \rightarrow\{1, \ldots, s\}$ is determined by $a(x)=i \Leftrightarrow x \in A_{i}$. Let $E(f, \alpha)$ be the class of functions $g$ such that there exists a Lebesgue probability space $(Y, \mathcal{B}, q)$ and a family of positively and negatively nonsingular maps $\left\{T_{i}\right\}_{i=1}^{S}$ such that $g$ is the density of an absolutely continuous invariant measure (a.c.i.m.) under the skew product as in (1).

We also denote by $\widehat{\alpha}$ the field of unions of elements of $\alpha$.

Definition 1. The process $(f, \alpha)$ is

(i) a Bernoulli process if for every $g \in E(f, \alpha), g$ is measurable with respect to $\mathcal{B}$,

(ii) a Markov process if for every $g \in E(f, \alpha), g$ is measurable with respect to $\widehat{\alpha} \times \mathcal{B}$.

Reduction of the condition in (ii) allows us to introduce quasi-Markovian processes.

Definition 2. We say that $(f, \alpha)$ is a quasi-Markovian process (q.m.p.) if for every $g \in E(f, \alpha)$ the set $\{g>0\}$ belongs to $\widehat{\alpha} \times \mathcal{B}$.

Some generalizations of Definition 2 and its applications can be found in $[1,2]$. By using Definitions 1 and 2 we obtain the following facts.

THEOREM 1. If $\alpha$ has the Markov property (i.e. $f(\alpha) \subset \widehat{\alpha}$ ) and if $\left(f^{n}, \bigvee_{i=0}^{n-1} f^{-i} \alpha\right)$ is a q.m.p. for some $n$ with respect to $m$ then $(f, \alpha)$ is also a q.m.p.

Theorem 2. If $X=[0,1], f$ is piecewise linear, $\alpha$ has the Markov property and $f \mid A_{i}$ is linear for $i=1, \ldots, s$ then $(f, \alpha)$ is a Markov process for every f-invariant probability measure which is absolutely continuous with respect to the Lebesgue measure. 
Let $\left(X_{n}\right)_{n=0}^{\infty}$ be a stationary process with $s$ states. Moreover, let $m$ be the measure on $\{1, \ldots, s\}^{N}$ determined by the finite-dimensional distributions of $\left(X_{n}\right)_{n=0}^{\infty}$.

Definition 3. We say that $\left(X_{n}\right)_{n=0}^{\infty}$ is a $k$-step Markov chain if

$$
\begin{aligned}
m\left(\omega_{n+1}=j \mid \omega_{0}=i_{0}\right. & \left., \ldots, \omega_{n}=i_{n}\right) \\
& =m\left(\omega_{n+1}=j \mid \omega_{n-k+1}=i_{n-k+1}, \ldots, \omega_{n}=i_{n}\right) .
\end{aligned}
$$

A 1-step Markov chain is a Markov chain. As a corollary to Theorem 1 we get

THEOREM $1^{\prime}$. If a stationary process $\left(X_{n}\right)_{n=0}^{\infty}$ is a $k$-step Markov chain for some $k \geq 1$ then it is a q.m.p.

Concerning Theorem 2 let us remark that if $f$ is not piecewise linear but piecewise monotonic then $(f, \alpha)$ is not a Markov process in general. However, in many cases it turns out to be a q.m.p., for example if $f$ is a Lasota-Yorke or Misiurewicz map ([4]). Let us turn to Theorem $1^{\prime}$. If we replace the Markov chain conditions by the Chapman-Kolmogorov equation (for the definition see below) then we may fall outside the class of quasi-Markovian processes (see Section 3).

Definition 4. We say that a process $(f, \alpha)$ satisfies the $C$-K-equation if

$$
m\left(f^{-n} A_{j} \mid A_{i}\right)=\left(\Pi^{n}\right)_{i j} \quad \text { for } i, j=1, \ldots, s \text { and } n \in \mathbb{N} .
$$

Here $\Pi_{i j}=m\left(f^{-1} A_{j} \mid A_{i}\right)$ for $i, j=1, \ldots, s$.

1. Proof of Theorem 1. We will use the skew product description of processes. Let us consider the skew product map

$$
T(x, y)=\left(f(x), T_{a(x)}(y)\right)
$$

where $a: X \rightarrow \alpha$ is determined by $a(x)=A \Leftrightarrow x \in A$. Here $\left\{T_{A}\right\}_{A \in \alpha}$ is a family of positively and negatively nonsingular maps of $Y$ into $Y$. Let $\nu$ be a $T$-invariant measure absolutely continuous with respect to $m \times q$. The measure $\nu$ is also $T^{n}$-invariant. Here

$$
T^{n}(x, y)=\left(f^{n}(x), T_{b(x)}(y)\right)
$$

where $b: X \rightarrow \beta=\bigvee_{i=0}^{n-1} f^{-i} \alpha$ is defined similarly to $a$. Here

$$
T_{B}(y)=T_{A_{n}} \circ \cdots \circ T_{A_{1}}(y) \quad \text { for } B=A_{1} \cap f^{-1}\left(A_{2}\right) \cap \cdots \cap f^{-(n-1)}\left(A_{n}\right) \text {. }
$$

Therefore,

$$
\left\{\frac{d \nu}{d(m \times q)}>0\right\}=\bigcup_{B \in \beta} B \times D_{B}
$$


if $\left(f^{n}, m, \beta\right)$ is a q.m.p. by Definition 2. From

we get

$$
T^{n}\left(\bigcup_{B \in \beta} B \times D_{B}\right)=\bigcup_{B \in \beta} B \times D_{B}
$$

$$
\bigcup_{B \in \beta} B \times D_{B}=\bigcup_{B \in \beta} f^{n}(B) \times T_{B}\left(D_{B}\right)=\bigcup_{A \in \alpha} A \times C_{A} .
$$

Here we use $f^{n}(B) \in \widehat{\alpha}$ for $B \in \beta$. Therefore $(f, m, \alpha)$ is a q.m.p.

2. Proof of Theorem 2. Consider the skew product map

$$
T(x, y)=\left(f(x), T_{a(x)}(y)\right)
$$

as in (1). Let $P$ be the Frobenius-Perron (F-P) operator for $T$, i.e.

$$
\int_{T^{-n} E} G d(\lambda \times q)=\int_{E} P(G) d(\lambda \times q)
$$

for $E \in \mathcal{A} \times \mathcal{B}$ and $G \in L_{1}(\lambda \times q)$. Here $\lambda$ is the Lebesgue measure. By the definition of $T$ we have

$$
P(w h)(x, y)=\sum_{i=1}^{s} a_{i} w\left(f_{i}^{-1}(x)\right) 1_{f\left(A_{i}\right)}(x) P_{i} h(y) \quad \text { for } w \in L_{1}(\lambda), h \in L_{1}(q),
$$

where $f_{i}=f \mid A_{i}, a_{i}=1 / f_{i}^{\prime}(x)$ for $x \in A_{i}$ and $P_{i}$ denotes the F-P operator for $T_{i}, i=1, \ldots, s$. Let $\mu$ be $f$-invariant, $\mu \ll \lambda$. Moreover, let $\nu$ be $T$-invariant and $\nu \ll \mu \times q$. Then also $\nu \ll \lambda \times q$ and therefore for $G=d \nu / d(\lambda \times q)$ we obtain $P^{n} G=G$ for $n=1,2, \ldots$ Let $A_{i_{1}, \ldots, i_{n}}=A_{i_{1}} \cap f^{-1}\left(A_{i_{2}}\right) \cap \cdots \cap$ $f^{-(n-1)}\left(A_{i_{n}}\right)$. Thus, as $f(\alpha) \subset \widehat{\alpha}$,

Consequently,

$$
P\left(1_{A_{i_{1} \cdots i_{n}}} h\right)=a_{i_{1}} 1_{A_{i_{2} \cdots i_{n}}} P_{i_{1}} h .
$$

$$
P^{n}\left(1_{A_{i_{1} \cdots i_{n}}} h\right)=\sum_{i=1}^{s} 1_{A_{i}} h_{i}
$$

By using approximation arguments as in [5] we conclude that

$$
G=\sum_{i=1}^{s} 1_{A_{i}} g_{i} \text {. }
$$

By repeating a similar reasoning for $\mu$ and the F-P operator for $f$ we get

$$
\frac{d \mu}{d \lambda}=\sum_{i=1}^{s} c_{i} 1_{A_{i}}
$$

Hence,

$$
\frac{d \nu}{d(\mu \times \lambda)}=\frac{d \nu}{d(\lambda \times q)}\left(\frac{d(\mu \times q)}{d(\lambda \times q)}\right)^{-1}=\sum_{i=1}^{s} 1_{A_{i}} d_{i} .
$$

Therefore $(f, \alpha)$ is a Markov process with respect to $\mu$. 
Let us remark that Theorem 3 in [7] and Theorem 6.3 in [8] are special cases of Theorem 2. Here the Markov property of $(f, \alpha)$ has been obtained for some classes of piecewise linear transformations by using an explicit definition of invariant measure.

3. C-K-process which is not a q.m.p. We recall the construction of Courbage and Hamdan from [3], using their notation. Let $K=\{0, \ldots, k-1\}$ and $\Omega=K^{N}=\{\omega: \omega(i) \in K, i=0,1,2, \ldots\}$. We say that a probability measure $\mu$ on $K^{n+1}$ is invariant if for any subsets $A_{1}, \ldots, A_{n}$ of $K$,

$$
\mu\left(K \times A_{1} \times \cdots \times A_{n}\right)=\mu\left(A_{1} \times \cdots \times A_{n} \times K\right) .
$$

Denote by $\operatorname{Inv}\left(K^{n+1}\right)$ the set of invariant measures. For $\mu \in \operatorname{Inv}\left(K^{n+1}\right)$ we define the measure $\nu_{0}=\Phi(\mu)$ on $\Omega$ by

$$
\begin{aligned}
\nu_{0}(\{\omega & \left.\left.\in \Omega: \omega_{0}=x_{0}, \ldots, \omega_{p n}=x_{p n}\right\}\right) \\
& =\mu\left(x_{0}, \ldots, x_{n}\right) \mu\left(x_{n+1}, \ldots, x_{2 n} \mid x_{n}\right) \cdots \mu\left(x_{(p-1) n+1}, \ldots, x_{p n} \mid x_{(p-1) n}\right)
\end{aligned}
$$

for all $p \geq 1$ and $x=\left(x_{i}\right)_{i=0}^{p n} \in K^{n+1}$. The measure $\nu_{0}$ is $\sigma^{n}$-invariant where $\sigma$ is the 1 -sided shift on $\Omega$. Now, we proceed to determine a suitable measure $\mu \in \operatorname{Inv}\left(K^{n+1}\right)$. Let $\Pi$ be a $k \times k$ stochastic strictly positive matrix and $\vec{p}$ be a row probability vector which is invariant under $\Pi$. By analyzing the proof of $\left[4\right.$, Theorem 3.1] we conclude that there exists a measure $\mu \in \operatorname{Inv}\left(K^{n+1}\right)$, for some prime number $n \geq 3$, such that

$$
\mu\left(A_{0} \times \cdots \times A_{n}\right)>0
$$

for any $A_{0}, \ldots, A_{n} \subseteq K$. The above holds because $\Pi$ is strictly positive and $\left.\mu\right|_{K^{n}}=\left.\mu_{\Pi}\right|_{K^{n}}$ (by conditions (3.4), (3.5) from [4]). Here $\mu_{\Pi}$ denotes the $(\Pi, \vec{p})$ Markovian measure. The measure

$$
\nu=\frac{1}{n} \sum_{i=0}^{n-1} \sigma^{i} \nu_{0},
$$

where $\nu_{0}=\Phi(\mu)$, is not $(\Pi, \vec{p})$ Markovian since $\mu \neq\left.\mu_{\Pi}\right|_{K^{n+1}}$ (by [4, $(3.18)])$. Moreover the process $(\sigma, \nu, \mathcal{P})$ satisfies the $\mathrm{C}$-K-equation with $\Pi$, i.e. $\nu\left(\sigma^{-n} P_{j} \mid P_{i}\right)=\left(\Pi^{n}\right)_{i, j}$ for $n=1,2, \ldots$, by $[4,(3.6)]$ and by the definition of $\nu$. Here $P_{i}=\{\omega \in \Omega: \omega(0)=i\}$. We will prove that $(\sigma . \nu, \mathcal{P})$ is not a q.m.p. To this end we show that there exists a Borel invariant set $E \subset \Omega$ of measure one such that $(E, \nu, \sigma)$ is positively nonsingular and $\nu\left(\sigma\left(P_{i} \cap E\right)\right)=1$ for $i=0, \ldots, k-1$. Combining this with the ergodic properties of $(\sigma, \nu)$ we get the desired conclusion. Let $\mathcal{P}_{0}^{n}=\bigvee_{i=0}^{n-1} \sigma^{-i} \mathcal{P}$. By the definition of $\nu_{0}$ and by (2) we get

LemMa 1. The process $\left(\sigma^{n}, \sigma^{i} \nu_{0}\right)$ is a Markov chain with vector $\vec{q}$ :

$$
\vec{q}_{\left(x_{0}, \ldots, x_{n-1}\right)}=\mu\left(x_{0}, \ldots, x_{n-i}\right) \mu\left(x_{n-i+1}, \ldots, x_{n-1} \mid x_{n-i}\right)
$$


and the strictly positive matrix $W$ :

$$
\begin{aligned}
& W_{\left(x_{0}, \ldots, x_{n-1}\right),\left(y_{0}, \ldots, y_{n-1}\right)} \\
& \quad=\mu\left(y_{0}, \ldots, y_{n-i} \mid x_{n-i}, \ldots, x_{n-1}\right) \mu\left(y_{n-i+1}, \ldots, y_{n-1} \mid y_{n-i}\right),
\end{aligned}
$$

for $i=2, \ldots, n$.

The case $i=1$ is considered in [3]. Here $\vec{q}_{\left(x_{0}, \ldots, x_{n-1}\right)}=\mu\left(x_{0}, \ldots, x_{n-1}\right)$ and

$$
W_{\left(x_{0}, \ldots, x_{n-1}\right),\left(y_{0}, \ldots, y_{n-1}\right)}=\mu\left(y_{0}, \ldots, y_{n-1} \mid x_{n-1}\right) .
$$

By [6, Chapter 10] there exists an invariant set $\Omega^{\prime} \subset \Omega$ with $\nu\left(\Omega^{\prime}\right)=1$ such that $\sigma \mid \Omega^{\prime}$ is positive nonsingular. The Markov chains $\left(\sigma^{n}, \sigma^{i} \nu_{0}\right)$ are ergodic, being aperiodic, for $i=1, \ldots, n$. Therefore the supports of $\sigma^{i} \nu_{0}$ are pairwise disjoint sets with respect to $\nu$. Hence there exists a Borel set $E_{0} \subset \Omega^{\prime}$ such that $\sigma^{n}\left(E_{0}\right)=E_{0}=\sigma^{-n}\left(E_{0}\right), \nu_{0}\left(E_{0}\right)=1$, the sets $\sigma^{-i} E_{0}$ are pairwise disjoint and $\sigma^{i} \nu_{0}\left(\sigma^{i}\left(E_{0}\right)\right)=1$ for $i=1, \ldots, n$. Set $\mu_{i}=\sigma^{i} \nu_{0}$ and $E_{i}=\sigma^{i} E_{0}, i=0, \ldots, n-1$. Let $E=\bigcup_{i=0}^{n-1} E_{i}$. Then $\sigma^{-1} E=E$ and $\nu(E)=1$.

REMARK. $(\sigma, \nu)$ is not totally ergodic as $\sigma^{n}\left(E_{0}\right)=E_{0}$ and $\nu\left(E_{0}\right)=1 / n$.

Obviously $(\sigma, \nu)$ is positively nonsingular on $E$.

Lemma 2. The partition $\mathcal{P}_{E}=\left\{P_{i} \cap E\right\}_{i=0}^{k-1}$ of $E$ is a Markovian generator for $(\sigma, \nu)$. Moreover, $\nu\left(\sigma\left(P_{i} \cap E\right)\right)=1$ for $i=0, \ldots, k-1$.

Proof. It suffices to show that $\nu\left(\sigma\left(P_{j} \cap E\right)\right)=1$ for $j=0, \ldots, k-1$. By the definition of $\nu$,

$$
\nu\left(\sigma\left(P_{j} \cap E\right)\right)=\frac{1}{n} \sum_{i=1}^{n} \mu_{i}\left(\sigma\left(P_{j} \cap E_{i-1}\right)\right) .
$$

By (2) and Lemma $1, \mu_{i}\left(\sigma^{n}\left(\left[x_{1}, \ldots, x_{n-1}, j\right] \cap E_{i}\right)\right)=1$ for every block $\left[x_{1}, \ldots, x_{n-1}\right]$. Hence the inclusion

$$
\sigma^{n-1}\left(\left[x_{1}, \ldots, x_{n-1}, j\right] \cap E_{i}\right) \subset P_{j} \cap E_{(i+n-1) \bmod n}=P_{j} \cap E_{i-1}
$$

implies $\mu_{i}\left(\sigma\left(P_{j} \cap E_{i-1}\right)\right)=1$. Hence $\mu_{i}\left(\sigma\left(P_{j} \cap E_{i-1}\right)\right)=1$ for $i=1, \ldots, n$. This finishes the proof.

Theorem 3. The process $\left(\sigma, \nu, \mathcal{P}_{E}\right)$ is not a q.m.p.

Proof. By the construction $(\sigma, \nu)$ is ergodic but not weakly mixing. The previous observations imply $\sigma \mid E$ is positively nonsingular and $\mathcal{P}$ is a Markovian generator for $(\sigma, \nu)$. Let $\mathcal{P}_{\sigma}$ denote the smallest field which contains $\left\{\sigma\left(P_{i} \cap E\right)\right\}_{i=0}^{k-1}$. By Lemma $2, \mathcal{P}_{\sigma}=\{\emptyset, \Omega\}$. Assume that $\left(\sigma, \nu, \mathcal{P}_{E}\right)$ is a q.m.p. Then, by [4, Lemma 2], all eigenfunctions of $\sigma$ are $\mathcal{P}_{\sigma}$-measurable, which implies that $(\sigma, \nu)$ is weakly mixing. This contradicts our assumption. 


\section{References}

[1] J. Aaronson and M. Denker, Local limit theorems for Gibbs-Markov maps, Stoch. Dyn. 1 (2001), 193-237.

[2] J. Aaronson, M. Denker, O. Sarig and R. Zweimüller, Aperiodicity of cocycles and conditional local limit theorems, ibid. 4 (2004), 31-62.

[3] M. Courbage and D. Hamdan, An ergodic Markov chain is not determined by its two-dimensional marginal laws, Statist. Probab. Lett. 37 (1998), 35-40.

[4] Z. S. Kowalski, Quasi-Markovian transformations, Ergodic Theory Dynam. Systems 17 (1997), 885-897.

[5] T. Morita, Deterministic version lemmas in ergodic theory of random dynamical systems, Hiroshima Math. J. 18 (1988), 15-29.

[6] W. Parry, Entropy and Generators in Ergodic Theory, Benjamin, New York, 1969.

[7] I. Shiokawa, Ergodic properties of piecewise linear transformations, Proc. Japan Acad. 46 (1970), 1122-1125.

[8] K. M. Wilkinson, Ergodic properties of a class of piecewise linear transformations, Z. Wahrsch. Verw. Gebiete 31 (1975), 303-328.

Zbigniew S. Kowalski

Institute of Mathematics and Informatics

Wrocław University of Technology

Wybrzeże St. Wyspiańskiego 27

50-370 Wrocław, Poland

E-mail: kowalski@pwr.wroc.pl

Received July 20, 2006;

received in final form February 23, 2007 